\title{
Eruptive syringomas in the groin
}

\section{Ivan V. Litvinov MD PhD, Fatemeh Jafarian MD}

Competing interests: None declared.

This article has been peer reviewed.

Affiliation: Division of Dermatology, McGill University Health Centre, Montréal, Que.

Correspondence to: fatemeh.jafarian@mcgill.ca

CMAJ 2014. DOI:10.1503 /cmaj.130793 Fatemeh Jafarian,

1 51-year-old man presented with a 6month history of asymptomatic eruptive skin-coloured lesions in his groin (Figure 1). He did not report any high-risk sexual behaviour leading up to development of the lesions. He had received a kidney transplant 3 years earlier and was taking a number of immunomodulating medications. Considering his immunosuppressed status, our differential diagnosis included atypical molluscum contagiosum, atypical genital warts, bowenoid papulosis and benign adnexal tumours. A shave biopsy showed tortuous ductal proliferation with occasional "comma-like tail" structures in the dermis (Figure 1), which is consistent with syringomas.

Syringomas are benign adnexal neoplasms of presumed eccrine or apocrine duct origin that affect about $1 \%$ of the population. ${ }^{1}$ They occur most commonly in women and usually present as soft, skin-coloured to slightly yellow papules on the lower eyelids and upper cheeks. ${ }^{1}$ Rarely, syringomas can occur in extraocular sites, including the neck, axilla, breasts, lower abdomen, thighs and groin. ${ }^{2}$ The risk factors for

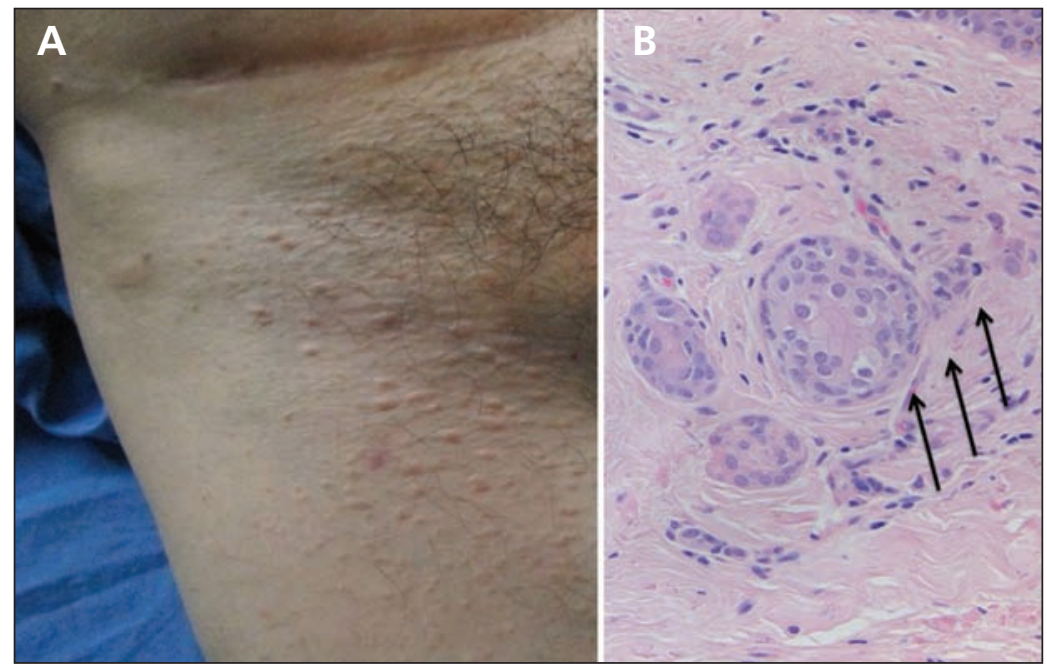

Figure 1: (A) Clinical presentation of eruptive syringomas in the groin of a 51 year-old man taking immunomodulating agents. (B) Histopathologic analysis of syringomas reveals tortuous sweat ducts in the dermis (arrows). syringomas are unknown. Some investigators have suggested that syringomas may be caused by hormonal factors ${ }^{3}$ or may be a reactive response to friction. ${ }^{4}$ Because of lifelong immunosuppression, transplant recipients are at greater risk of syringomatous carcinoma, a rare malignant variant, which may have a similar presentation to syringomas. ${ }^{5}$ However, no specific association between syringomas and organ transplantation has been established.

Syringomas may be distinguished clinically from xanthelasmas, verrucae or carcinomas because they are monomorphic and evenly distributed; the diagnosis can be confirmed by biopsy in uncertain cases. On histologic examination, syringomas are easily recognizable by the presence of comma- or tadpole tail-shaped sweat ducts in the dermis. ${ }^{2}$

Even though syringoma lesions are benign, they may be cosmetically distressing for patients. Treatment options, mostly based on small case series and case reports, include surgical excision, electrodessication and curettage, chemical peels, cryosurgery and laser therapy. ${ }^{1,2}$ However, because these tumours are embedded deep in the dermis, all treatments are associated with substantial risk of recurrence and may lead to scarring and pigment changes. ${ }^{1,2}$ Given the benign nature of this condition, our patient declined cosmetic treatment and is being followed in our solid-organ transplant clinic for signs of any other skin malignancies or infection.

\section{References}

1. Soler-Carrillo J, Estrach T, Mascaró JM. Eruptive syringoma: 27 new cases and review of the literature. $J$ Eur Acad Dermatol Venereol 2001;15:242-6.

2. Petersson F, Mjornberg PA, Kazakov DV, et al. Eruptive syringoma of the penis. A report of 2 cases and a review of the literature. Am J Dermatopathol 2009;31:436-8.

3. Bal N, Aslan E, Kayaselcuk F, et al. Vulvar syringoma aggravated by pregnancy. Pathol Oncol Res 2003;9:196-7.

4. García-Salces I. Syringomas and lichen amyloidosus as a presentation of reactive dermatosis [article in Spanish]. Actas dermosifiliograficas. 2009;100:719-21.

5. Hoppenreijs VP, Reuser TT, Mooy CM, et al. Syringomatous carcinoma of the eyelid and orbit: a clinical and histopathological challenge. Br J Ophthalmol 1997;81:668-72. 